\title{
DESIGNING NO-SQL BASED PERSONAL HEALTHCARE DATA EXCHANGE SCHEMA
}

\author{
Hee Joung Hwang \\ Dept. of Computer Engineering, Gachon University. \\ 1342 Seongnamdaero, Seongnam-si, Kyeonggi-do, Korea
}

\begin{abstract}
With the advance of micro-service architecture, existing large systems have begun to be deployed as small service units. There is an increasing number of NoSQL-based services that can handle unstructured data in the massive schema structure of large RDBMSs. However, the heterogeneity and diversity of the numerous existing systems impede the well-informed selection of a data store appropriate for a given application context.

In this paper, we design a NoSQL - based data exchange server to integrate Healthcare data and implement Restful API for interworking with various medical data sources. Experimental results show that medical data such as HL7 FHIR, Fitbit and CDM can be exchanged.
\end{abstract}

\section{KEYWORDS}

Data Exchange Server, HIS, NoSQL, Healthcare

\section{INTRODUCTION}

Due to the rapid development of IT technology in recent years, the amount of data has increased exponentially. As the penetration rate of smartphones increases and the number of wearable devices and IoT technology increases, the amount of data to process has also increased dramatically and the importance of storage and processing power has also increased. In the past, relational database management systems (RDBMS), such as MySQL, Oracle, and Postgre SQL, have begun to show limitations in terms of cost and efficiency when processing large amounts of data, effectively managing and processing large amounts of data [Abadi, 2012]. NoSQL is growing rapidly, although Oracle, MySQL and MSSQL are still at the top of database rankings [Choi et al, 2016].

In this study, we designed and implemented a Health Data Exchange Server architecture that works with various healthcare data sources. Generally, standards such as HL7, HL7 CDA, and HL7 FHIR are required for medical information systems and medical data exchange. However, methods other than HL7 FHIR were expensive to implement with too large a specification, so the standard did not spread.

Our works is theoretically compatible with systems such as HL7 v 2.x, CDA (Clinical Document Architecture) / CCD [Zhang et al, 2015], ASTM CCR [Tseng et al, 2003] and HL7 MLLP, IHE XDS.b. Outbound modules supporting standards-based communication protocols have been developed and can be interchanged with existing medical data systems to ensure interoperability with a variety of medical devices.

In particular, the development of medical and personal healthcare devices and the increase in personal health data require a repository structure to effectively integrate and operate these data.

\section{DESIGN AND IMPLEMENTATION}

\subsection{Data Message Schema}

There are two main models of data architecture for health information exchange. One is a federated or distributed model, and the second is a centralized model. There is also a hybrid model with two elements. The 
centralized HIE has a central (or master) database that holds a complete copy of all records for all patients included in the HIE. The federated HIE does not have a master database.

In the federated model, each health care provider must maintain a record of individual patients. The main function of the HIE in this model is to enable the provider to exchange patient records as needed. For example, when a doctor in the federal HIE requests a record for patient $\mathrm{Y}$, a query is sent to each server in the system to request that records for patient $\mathrm{Y}$ be returned. Each federated HIE can do this in a slightly different way. The notable difference is that the affiliate model does not have a central database to store and download previously compiled medical records.

In personal healthcare areas there are various types of data exist like HL7 FHIR, ISO, and OHDSI. Among them, HL7 FHIR is attracting attention because it uses flexible message standards such as JSON and XML. However, server systems that handle these messages require a micro-services architecture based on modular software architecture, unstructured data processing, and open APIs.

Figure 1 shows 3 different personal healthcare data.



(a) HL7 FHIR

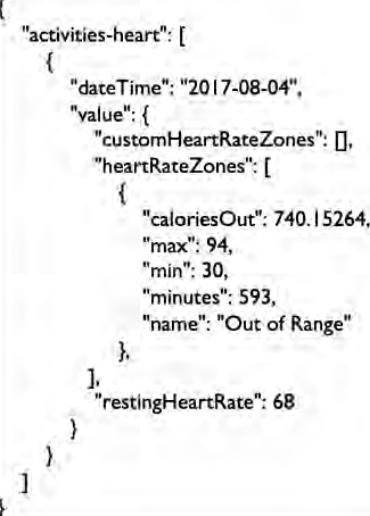

"activities-heart":

i

"dateTime": "2017-08-04",

"value": \{

"customHeartRateZones": [

"heartRateZones": [

\{

"caloriesOut": 740.15264.

"max": 94,

"min": 30,

"minutes": 593 ,

1, .

(b) Fitbit smart band



\{

"DAYS SUPPLY DISTRIBUTION": ( "CATEGORY": "Days supply". "MIN_VALUE": 10 "PIO_VALUE": 10, "P25_VALUE": 30, "MEDIAN_VALUE": 30. "P75_VALUE": 30, "MAX_VALUE": 30 "QUANTITY_DISTRIBUTION": \{ "CATEGORY": "Quantity". "MIN_VALUE": 0 , "PIO_VALUE": 10 "P25_VALUE": 30 , "MEDIAN VALUE"; 30 "P75 VALUE": 60, "MAX̄_VALUE": 120 \},

(c) OHDSI CDM

Figure 1. Personal healthcare data schema

\subsection{Data Repository}

NoSQL systems are purpose-built solutions, designed to address specific technical requirements. NoSQL systems originated to provide high throughput, fault-tolerant horizontally scalable simple data storage and retrieval with a bare minimum of additional functionality. Specifically, NoSQL systems were created in order to provide:

Horizontally distributed data management of simple structured and unstructured data across a large cluster of commodity storage systems,

Highly fault-tolerant data management and ability to continue operating even after multiple hardware and system failures,

- Very high throughput for simple read/write operations, with limited or no transaction semantics,

- Schema-less or flexible schema definitions allowing highly variable data and record structures,

- Application and application developer-centric special purpose data models and APIs

The most commonly employed distinction between NoSQL databases is the way they store and allow access to data. Each system covered in this paper can be categorized as either key value store, document store or wide-column store. 


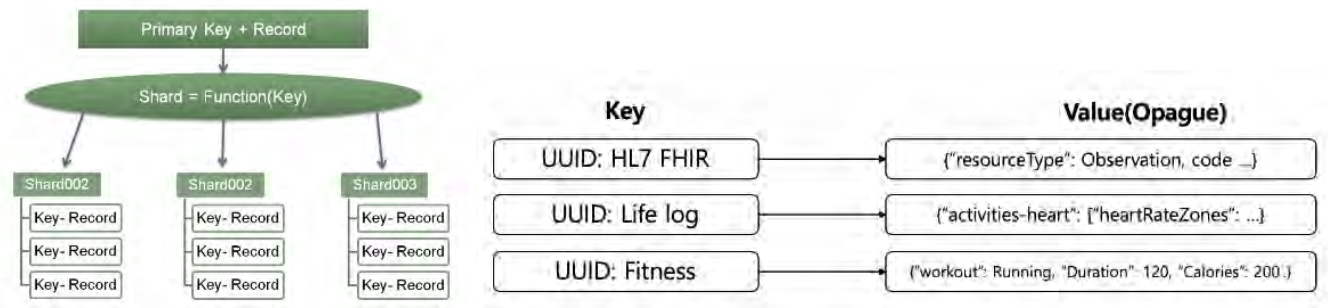

Figure 2. Proposed NoSQL repository data structure

\section{CONCLUSION}

NoSQL database technology is here to stay. It addresses specific technical requirements that are not as efficiently or cost effectively addressed with other data management technologies, including RDBMS systems. The RDBMS and NoSQL Database are complementary in the sense that they work together to address the overall data management needs of our customers, each providing the technical capabilities required by today's complex and evolving applications.

In summary, we are confident that the proposed schema model is an effective decision to support effective filtering. Centralized large NoSQL database system Requirements. NoSQL Toolbox Mapping between functional and non-functional requirements A common implementation technique for classifying constantly evolving NoSQL spaces.

\section{ACKNOWLEDGEMENT}

This research was supported by the MSIT(Ministry of Science and ICT), Korea, under the ITRC (Information Technology Research Center) support program(IITP-2019-2017-0-01630) supervised by the IITP (Institute for Information \& communications Technology Promotion)

\section{REFERENCES}

Abadi D (2012) Consistency tradeoffs in modern distributed database system design: cap is only part of the story. Computer 45(2):37-422. Attiya H, Bar-N.

Choi, J., Choi, C., Ko, H., and Kim, P., "Intelligent Healthcare Service Using Health Lifelog Analysis,” Journal of medical systems, 40(8), 1-10, 2016.

Minkyung Kim, Dong-Wook Lee, Kangseok Kim, Jai-Hoon Kim, "Hierarchical struc- tured data logging system for effective lifelog management in ubiquitous environment," Multimedia Tools and Applications, 2013.

Myung-Kyu Yi and Hee-Joung Hwang," A New Lifelog Management Scheme for In- telligent Healthcare Service”, International Journal of Elderly Welfare Promotion and Management, Vol. 1, No. 1, pp. 29-36, 2017.

Reza Rawassizadeh, Martin Tomitsch, Katarzyna Wac, A. Min Tjoa, "UbiqLog: a generic mobile phone-based life-log framework," Personal and Ubiquitous Computing, Vol. 17(4), pp. 621-637, 2013.

Sheldon M. Ross, "Introduction to Probability Models: Eighth Edition," Academic Press, 2002.

Wiese L et al (2015) Advanced Data Management: For SQL. Cloud and Distributed Databases. Walter de Gruyter.

X. Zhang, J. Castellanos, and A. Campbell, "Design and Performance of Mobile IP Paging," ACM Mobile Networks and Applications, pp.127-141, March, 2002.

Yu-Chee Tseng, Lien-Wu Chen, Ming-Hour Yang, Jan-Jan Wu, “A stop-or-move mo- bility model for PCS networks and its location-tracking strategies," Journal of Com- puter Communication, pp. 1288-1301, 2003.

Zhang H, Chen $\mathrm{G}$ et al (2015) In-memory big data management and processing: a survey. TKDE. 